\title{
Las Creencias de los profesores rurales: una tarea pendiente para la investigación educativa
}

\author{
Rural Teacher's beliefs: a pending task for educational research
}

As Crenças dos professores rurais: uma tarefa pendente para a investigação educativa

\author{
Daniela Vera Bachmann ${ }^{1}$, Sonia Osses ${ }^{2}$, \\ Ernesto Schiefelbein Fuenzalida ${ }^{3}$ \\ ${ }^{1}$ Universidad Austral de Chile, Escuela de Psicología, Doctorado en Ciencias de la Educación, Facultad de \\ Educación y Humanidades, Universidad de La Frontera, Temuco, Chile, danielavera@spm.uach.cl \\ ${ }^{2}$ Universidad de La Frontera, Doctora, Facultad de Educación y Humanidades, Departamento de Educación, \\ Casilla 54-D, Temuco, Chile, sosses@ufro.cl \\ ${ }^{3}$ Universidad de La Frontera, Facultad de Educación y Humanidades, Departamento de Educación, Temuco, \\ Chile, pschiefe@gmail.com
}

\begin{abstract}
RESUMEN
Las investigaciones sobre las creencias en el proceso de enseñanza-aprendizaje se han convertido en el último tiempo en un tema de gran preocupación, debido a la creciente evidencia que señala que las creencias tienen un fuerte impacto en el quehacer pedagógico de los profesores. Pese a esto, se ha demostrado que las investigaciones realizadas en este ámbito se han centrado, preferentemente, en el sistema educativo de zonas urbanas, en niveles de enseñanza básica, media, y educación superior, en desmedro de lo que ocurre en la educación que se desarrolla en las zonas rurales de nuestro país, sector que históricamente ha mostrado los resultados educativos más bajos. En este artículo se desarrolla una visión que permitiría hipotetizar, desde el estudio de las creencias, una de las posibles variables personales del profesor para explicar los resultados de aprendizaje en la educación rural de nuestro país. Se concluye la necesidad de investigar las creencias que sustentan los profesores rurales respecto del proceso educativo, dada su relevancia en el proceso de enseñanza y aprendizaje de los estudiantes.
\end{abstract}

Palabras clave: educación rural, creencias, práctica pedagógica.

\begin{abstract}
Research on the beliefs in the teaching-learning process has recently become a great issue due to the increasing evidence that points out that it has a great impact in the teacher's pedagogical activities. Nevertheless, it has become evident that research on this matter has been focused mainly on the educative system performed in urban areas, on basic and high school levels and higher education, underestimating what occurs in rural areas of our country, field that historically has shown the lowest academic performance. This article presents a view that allows making hypothesis, from the study of the beliefs, of the possible personal variables of the teacher that could explain the results of the learning process shown in the rural education of our country. As conclusion, it is necessary to do research on the rural teacher's beliefs, from the educational perspective, because of the direct consequences on the learning process of the students.
\end{abstract}

Key words: rural education, beliefs, pedagogical practice.

\section{RESUMO}

Considerado o processo de ensino-aprendizagem na atualidade, as investigações sobre crenças converteram-se em tema de grande preocupação, dada à crescente evidência de que as crenças têm forte impacto no fazer pedagógico de professores. Apesar de isto, evidencia-se que as investigações realizadas neste âmbito, centraram-se preferencialmente no sistema educativo urbano, em níveis de Educação Básica, Ensino Médio e Educação Superior, ao contrário do que ocorre na educação do campo de nosso país, onde, historicamente, os resultados educativos sempre foram 
inferiores. Desenvolve-se uma visão que permite hipotetizar que, a partir do estudo das crenças, uma das possíveis variáveis identitárias do professor, possa explicar os resultados de aprendizagem na educação do campo de nosso país. Conclui-se que há necessidade de pesquisas relacionadas às crenças que sustentam os professores rurais em relação ao processo educativo, dada a relevância disso no processo de ensino-aprendizagem dos estudantes desses locais.

Palavras chave: educação do campo, crenças, prática pedagógica.

\section{INTRODUCCIÓN}

La educación básica en el contexto rural se ha definido por su localización, el carácter cultural de la población escolar que atiende y sus especificidades pedagógicas (Williamson, 2004). Dadas principalmente por las dinámicas de vida propias de sus habitantes, marcan un importante espacio dentro de la cultura del país y una singular forma de ver el mundo y entender su entorno (Cox, 2003), las cuales requieren de una educación que dé respuesta a sus particularidades. Por este motivo, el sistema educativo chileno ha promovido el desarrollo de prácticas pedagógicas modernas, contextualizadas al mundo rural, con materiales específicos para la ruralidad y trabajo colaborativo entre los profesores. Los objetivos de estos cambios se han orientado a otorgar más y mejores aprendizajes para todos los niños y niñas y a subir los estándares de calidad para todas las escuelas rurales, mediante el mejoramiento de los procesos pedagógicos y de gestión de dichos establecimientos, a fin de lograr resultados de aprendizaje satisfactorios (Ministerio de Educación, 1998).

De acuerdo a lo anterior, para cumplir con tales objetivos se hace necesario considerar en el proceso de enseñanza y aprendizaje a sus actores, principalmente el protagonismo del profesor. Éste constituye uno de los actores más importantes del proceso educativo, en general, ya que participa activamente como mediador en el proceso de aprendizaje de los niños (Ministerio de Educación, 1999); de hecho, la calidad de la enseñanza se relaciona, entre otras variables, con las características personales y profesionales del profesor y con el modo en que enfrenta y conduce el proceso pedagógico (De Andraca y Gajardo, 1992).

En este punto es necesario destacar la emergencia de un creciente interés por conocer el pensamiento de los profesores, dado que se ha comprobado empíricamente que la postura de los profesores ante los procesos de innovación y mejoramiento educativo representa un fuerte condicionante, sino determinante del éxito de cualquier iniciativa en este sentido (De Miguel, 2001). Diversas investigaciones han buscado identificar y describir el pensamiento del profesor, los resultados han permitido conocer aspectos que en variadas ocasiones han sido ignorados y que tienen directa relación con el proceso de aprendizaje de los estudiantes (Delandshere y Jones, 1999; Marcelo, 2002; Torrence y Prior, 2001). Lamentablemente, ninguna de ellas da cuenta de lo que ocurre en la educación rural.

Lo anterior implica, por tanto, considerar la incorporación de las variables personales del profesor que influyen en su desempeño profesional e impactan en el proceso educativo, ya que sus concepciones sobre la educación, les llevarán a interpretar, decidir y actuar en la práctica; de ahí la importancia de estudiar estas variables -dentro de las cuales se encuentran las creencias- que resultan centrales al plasmar en la relación pedagógica, la visión que el profesor tiene de su trabajo, de los alumnos y el desempeño de éstos. Dicho aspecto se ha comprobado en el hecho de que algunos de los problemas inherentes 
al bajo rendimiento, dicen relación con el origen, formación y características personales del profesor (Rivera y Milicic, 2006).

En este sentido, las creencias han demostrado tener una significativa relación con el proceso educativo, evidenciando que las creencias que un profesor sustente respecto de éste ocuparán un lugar central en su estructura cognoscitiva, afectando lo que percibe e interpreta, transformándose en elemento constitutivo de actitudes, valores, ideologías y prejuicios, conceptos con gran relevancia en la explicación del comportamiento humano (Navas, Sampascual y Castellón, 1992; Myers, 2000).

De acuerdo a lo señalado, considerando la posición central que ocupan las creencias como determinantes del comportamiento, y dada la importancia del educador rural como uno de los principales protagonistas del proceso educativo y su rol determinante en la construcción de nuevas relaciones pedagógicas, el presente artículo pretende incentivar la investigación educativa sobre creencias en las zonas rurales de nuestro país, en donde los estudios en el área son escasos y los indicadores educativos son los más bajos del sistema educacional chileno.

\section{EL ENTORNO RURAL HOY}

Las primeras concepciones respecto del mundo rural impusieron por siglos la idea de un ambiente estable y con una función social determinada, que no debía cambiar. Esa noción tradicional impregnó hasta hace muy poco las imágenes relativas a lo rural, relacionándolo con organizaciones sociales agrarias o regiones de hábitat disperso (PNUD, 2008). Si bien esta visión del mundo rural aún en algunos casos es admitida y utilizada, lo cierto es que hoy no existe una concepción única para el mundo rural en su totalidad y para las diversas personas que lo habitan (San Miguel, 2005), debido principalmente a que en la actualidad el entorno rural ha cambiado.

Hoy, más que una estructura estable, la ruralidad constituye un proceso en constante dinamismo y movimiento, lo que desafía profundamente las perspectivas que se han usado habitualmente para definir y comprender lo rural (Canales, 2005). El Informe sobre Desarrollo Humano Rural en Chile (PNUD, 2008), muestra que la ruralidad de hoy no constituye una forma de vida y una visión de mundo totalmente opuesta o excluyente de las formas de vida y visiones de mundo de la sociedad en general. Hoy la ruralidad y las grandes urbes constituyen dos líneas paralelas y conectadas de una misma historia, en donde los avances en cuanto al acceso a la información y la comunicación han tornado difusos los límites entre un espacio y otro.

En este sentido, definir qué es la ruralidad hoy implica abandonar definiciones que por décadas formaron parte de las representaciones sociales del sistema chileno, en las cuales el mundo rural se asociaba mayoritariamente al agro, al campesinado, al retrazo y la pobreza (Williamson, 2004).

En nuestro país existen aproximadamente 290.000 familias asentadas en el medio rural constituidas por 2.185.831 personas. Encuestas nacionales muestran que el $26.0 \%$ de los hogares rurales, pese al desarrollo económico que ha experimentado el país, aún se ubica bajo la línea de la pobreza y que el 30.6\% de los habitantes del sector vive en esos márgenes. Estas carencias, al perjudicar especialmente las oportunidades de los 
niños y jóvenes de familias rurales de escasos recursos, llegan a convertirse en fuente de mayores desigualdades en el futuro próximo (Williamson, 2004).

Es esta complejidad dada por el entrelazamiento entre la pobreza y las oportunidades a las cuales pueden acceder los niños que viven en zonas rurales, la que plantea nuevos desafíos al mejoramiento de las condiciones y de la calidad de vida de los habitantes de zonas rurales, lo que se genera por medio de mejoras salariales reflejadas en aumentos productivos; es decir, se necesita que el mundo rural genere una mayor productividad para establecer una mejora en la calidad de vida de sus trabajadores. Ahora bien, se reconoce que esto sólo puede lograrse con acceso a educación de calidad, pues se ha demostrado que los agricultores con educación básica y media completa tienen más probabilidades de adoptar nuevas tecnologías y ser más productivos; de este modo están mejor formados para tomar decisiones, mejor informados para sus vidas y sus comunidades, como asimismo, para ser participantes activos en la promoción de las dimensiones económica, social y cultural del desarrollo (Atchoarena y Gasperini, 2004; Villarroel, 2004).

De esta forma, la educación adquiere un rol fundamental en el desarrollo humano y la mejora en la calidad de las personas que viven en el medio rural, dos aspectos que van de la mano con el acceso a oportunidades de crecimiento y de conocimiento, dos nodos que se hacen latentes en la manera en que se forman y se educan las personas que viven en ruralidad y en la calidad que dicha educación es puesta a disposición de la gente rural (Montero, 2001).

\section{EDUCACIÓN RURAL EN CHILE}

La educación en nuestro país constituye una de las principales bases de nuestra sociedad. Es un proceso considerado por la mayoría de los ciudadanos como la vía necesaria para adquirir habilidades y conocimientos de gran importancia para el desenvolvimiento dentro de los marcos y procesos sociales. Por este motivo, es de gran relevancia propiciar que cada uno de los educandos, independientemente de su clase social o entorno familiar, logre los objetivos de aprendizaje (Arancibia, Herrera y Strasser, 2000). Para cumplir con tal desafío en un mundo en permanente cambio y expansión, se requiere de reestructuraciones permanentes, con todo lo que ello significa en materia de renovación y conservación social (De Pujadas, 1991). El reconocimiento de esta situación ha implicado revisar profundamente el rol de la escuela, reconocer la complejidad de los procesos de transformación de los diversos contextos en donde ésta se inserta y del aprendizaje que en ella se desarrolla.

Debido a lo anterior, a partir de los años noventa, el Ministerio de Educación definió a la escuela rural como una de sus prioridades, asumiéndola como una institución de promoción de la igualdad de oportunidades para todos los niños y niñas de familias rurales y de contribución a la integración del mundo rural a la sociedad futura. El desafío ha sido y seguirá siendo salir del diagnóstico de inequidad y baja calidad para el sector educativo rural, asegurando a sus escolares igualdad de oportunidades en función de los resultados de sus estudios, considerando sus antecedentes de socialización, es decir, una educación diferenciada en los insumos y procesos, pero buscando el logro de resultados equivalentes a los esperados para todos los alumnos del país (San Miguel, 2000). 
En la actualidad, la red escolar cuenta con aproximadamente 5.000 establecimientos rurales de enseñanza básica. La distancia entre escuelas raramente excede los 10 kilómetros, muchas veces equivalente a la que existe entre las escuelas y el hogar de los niños matriculados en ellas (Williamson, 2004).

En el medio rural es predominante el sistema municipal de educación (81\%) en relación al particular subvencionado (18,4\%). Sólo en la Región de Los Lagos y en la Región de La Araucanía este último porcentaje es superior; en la primera corresponde a un $22,0 \%$ de establecimientos particulares subvencionados rurales (256 sobre un total de 1.163 rurales y 1.606 totales) y en la segunda alcanza un 44,0\% (405 de 920 establecimientos rurales en relación al total regional de 1.348) (Williamson, 2004; San Miguel, 2005).

En el contexto rural funcionan escuelas uni y bidocentes, tridocentes y polidocentes de distinto tamaño; grandes, medianas y pequeñas. Las primeras se concentran en las zonas más pobres y con menos posibilidades de desarrollo, en tanto las últimas funcionan en zonas donde la modernización del agro presiona por mayores y mejores logros de la escolarización (De Andraca y Gajardo, 1992; Williamson, 2004).

Este intento de perfil de la escuela rural hace suponer que, gracias a su diversidad y clasificación, favorece la experimentación educativa y el desarrollo de una pedagogía activa. De este modo, parece estar dotada de maestros "integrales" (a veces de maestros polivalentes), tener alumnos diversos, maestros que pueden impartir una enseñanza individualizada; contar con un sistema de enseñanza concéntrica y flexible y, por último, poseer un sistema de control "blando", respetuoso y mucho más educativo (Feu i Gelis, 2004).

En este contexto, el profesor se ve enfrentado a la necesidad de adecuarse al medio educativo rural y a las particularidades que éste presenta, debiendo adaptar sus prácticas pedagógicas a las condiciones en que desarrolla su trabajo y a las oportunidades que la educación en escuelas pequeñas parece presentar (Villarroel, 2003).

\section{EL ROL DEL PROFESOR RURAL}

En la actualidad, el rol que se atribuye a los profesores en los cambios organizativos y curriculares que continuamente experimenta el sistema educativo es fundamental, debido a que el funcionamiento de la educación se decide en última instancia, por la calidad y funcionalidad de las prácticas pedagógicas (Ávalos, 2003; González, 1999). De esta manera, los principales objetivos para el éxito del proceso de modernización de la educación se centran en la calidad de la enseñanza, es decir, en la acción y competencias del profesorado, las que se conciben como dirigidas hacia la obtención de objetivos específicos de aprendizaje, enseñanza y desarrollo (Sánchez, 2001).

Algunos estudios han demostrado que, para introducir cambios en el proceso educativo, es necesario incorporar como variable el protagonismo del profesor como un mediador de la interacción de cada alumno con los objetivos de conocimiento (Ávalos, 2003), ya que se ha evidenciado que, tanto la calidad de la enseñanza impartida por la escuela, como los resultados del aprendizaje, se relacionan entre otras variables, con el rol principal que juega el profesor en el proceso de enseñanza-aprendizaje, lo que considera la inclusión de sus características personales y profesionales y el modo con que éste enfrenta y conduce el proceso pedagógico (Fang, 1996; Pajares, 1992). 
Actualmente, existen variadas expectativas en relación con el profesor rural y su desempeño docente. Sin embargo, no hay definiciones únicas de lo que es o se espera de él. Lo que existe es un conglomerado heterogéneo de profesores con las más variadas características personales y trayectoria ocupacional. Sus condiciones de trabajo varían según el tipo de escuela en la que trabajan y las características de la zona en la que ésta se localiza. Aunque no existe un prototipo de profesor, es lícito pensar que el papel del profesor rural en el desarrollo local, es el de ser un facilitador del proceso de aprendizaje y un creador de espacios favorables para una auténtica participación comunitaria. El papel del profesor rural, por tanto, no se limita sólo a la relación profesor-alumno, pues su labor se concibe como un servicio a la comunidad (Villarroel, 2003; Thomas, 2005). Por este motivo, resulta posible esperar del educador rural una labor abierta, creativa, que en la medida de lo posible responda a requerimientos de su medio y no se ciña, exclusivamente, a satisfacer las exigencias de un programa de aplicación general (De Andraca y Gajardo, 1992).

Esta amplia gama de tareas que se espera debe cumplir el profesor rural, evidencia que se le ha exigido dar respuesta a múltiples demandas, lo que podría afectar en ocasiones, tanto la competencia como la dedicación y, muchas veces, incidir negativamente en la motivación para enseñar, factor crítico en la enseñanza efectiva (Schiefelbein, 1992; Thomas, 2005).

Lo anterior se ve agravado si se considera que la mayoría de los profesores rurales no recibe una formación específica para desempeñarse como profesor en el área rural, es decir, una formación que lo habilite para el desempeño de las funciones descritas, salvo en el caso de quienes han sido formados en la escuela de Pedagogía en Educación Básica con Mención en Educación Rural y Desarrollo, perteneciente a la Universidad de Playa Ancha, y en la Escuela de Pedagogía General Básica de la Pontificia Universidad Católica Sede Villarrica; se trata en su mayoría de profesores de enseñanza básica que han cursado la carrera en universidades donde no se habilita a los docentes para enfrentar la diversidad de problemas y situaciones que es posible encontrar en las zonas rurales (De Andraca y Gajardo, 1992). Esto trae como consecuencia que los profesores no se encuentren capacitados para interpretar las múltiples y complejas dimensiones de la realidad del mundo rural, que tampoco cuentan con la preparación necesaria para enfrentar los problemas pedagógicos que derivan de la situación de las escuelas en el campo, ni estén capacitados para relacionarse con los niños, sus familias y la comunidad rural en su conjunto (Villarroel, 2003).

Los nuevos desafíos y la multiplicidad de roles que demanda la docencia rural (Thomas, 2005), requieren de la consideración de variables personales del profesor que impactan en el proceso educativo. Ejemplo de esto lo constituyen las creencias que los profesores sustentan, las cuales resultan centrales, ya que se plasman en la relación pedagógica, incidiendo tanto en la visión que el profesor tiene de su trabajo como de sus alumnos y el desempeño de éstos (De Andraca y Gajardo, 1992; Villarroel, 2003).

La evidencia teórica señala la relación existente entre lo que el profesor cree respecto a sus alumnos y el rendimiento académico que éstos tienen, en tanto las actividades que los profesores realizan y el modo en que lo hacen se ve teñido por sus creencias, las cuales han sido construidas mediante las experiencias pasadas, generando así una cadena de prejuicios sobre la manera en que se dan, por ejemplo, los procesos evaluativos (Meckes, 2007). 
Myers (2000) plantea que las caracterizaciones que los profesores realizan de los estudiantes se correlacionan con los logros de éstos. Estudios realizados muestran que las expectativas de los profesores sobre el rendimiento de los alumnos repercuten en las calificaciones de éstos; los alumnos de los que se espera mejores resultados de hecho los obtienen, a pesar de que la expectativa muchas veces carece de fundamento (Duschatsky, 1999). Por lo tanto, la evidencia teórica avala el supuesto de que lo que el profesor cree de sus alumnos incide en el rendimiento de éstos, pues sus conductas están determinadas por sus creencias, las que a su vez, han sido construidas sobre los aspectos sociales y culturales de los educandos, quienes responden consecuentemente a las diferentes conductas, expectativas y creencias del profesor (Arancibia, Herrera y Strasser, 2000; Ministerio de Educación, 2003). Esto resulta particularmente sensible en las zonas rurales, en donde existen altos índices de vulnerabilidad y en donde los niños y niñas con bajas calificaciones, podrían llegar a pensar que el éxito en la escuela es un privilegio de algunos, sintiéndose excluidos de ella, lo que podría conducirlos, finalmente, al fracaso escolar y, eventualmente, a la deserción (Contreras y Prieto, 2008).

Lo mencionado demuestra, por tanto, que los resultados alcanzados por los estudiantes, no sólo serían un reflejo de sus capacidades, sino que también evidenciarían las concepciones sustentadas por los profesores acerca del proceso educativo a partir de las cuales deriva su quehacer pedagógico (Fang, 1996; Litwin, 2005).

\section{CREENCIAS}

Las creencias ocupan un lugar central en la estructura cognoscitiva de los individuos. Ellas afectan poderosamente la forma en que percibimos, interpretamos e, incluso, recordamos los acontecimientos. Las creencias constituyen verdaderas "teorías" acerca del mundo social y no social. Son consideradas como elementos constitutivos de diversos conceptos, tales como actitudes, valores, ideologías y prejuicios y éstos, a su vez, han demostrado una gran relevancia para la explicación del comportamiento humano (Myers, 2000).

Estas creencias están compuestas por un red de ideas interconectadas que se construyen a partir de experiencias que el sujeto vive cotidianamente. Son versiones simplificadas de la realidad que tienen organización interna, son estructuradas y sistemáticas, permitiendo a los individuos interpretar los acontecimientos y tomar decisiones respecto de los sucesos que ocurren. Las creencias tienen un origen de carácter cultural, en tanto se construyen en "formatos de interacción social y comunicativa" (Jackson, 2002).

Según lo planteado por Pajares (1992), las creencias se muestran inicialmente fragmentarias e inconexas, profundamente arraigadas en la experiencia, individual y cotidiana, las cuales en la medida que transcurre el tiempo se transforman, organizándose en estructuras que influyen en una variedad de procesos cognitivos, llegando a explicaciones relativamente coherentes entre sí, que en la interacción con la realidad se constituyen en representaciones, modelos que permiten al individuo que las posee, comprender, explicar y finalmente, predecir los eventos a su alrededor; se puede hablar de ellas con propiedad en términos de teorías implícitas que cada persona construye para dar sentido y consistencia a la experiencia cotidiana (Goodson y Numan, 2002; Hashweh, 2005; Rodrigo, Rodríguez y Marrero, 1993). 
La importancia de las creencias radica en que éstas se encuentran presentes en todos los individuos, manifestando claros efectos, ya que influyen en la forma en cómo éstos sienten y actúan, y al hacerlo así, generan su propia realidad (Myers, 2000). En el ámbito social e interpersonal, las creencias cumplen un papel fundamental, pudiendo establecer los fines de la acción individual y colectiva (González, 1999); además, algunas de éstas pueden ser compartidas por diferentes individuos, validándose por medio del acuerdo con otro, permaneciendo dentro de un marco de significados y supuestos socialmente compartidos, así como dentro de los medios, los modos y los principios socialmente establecidos de la comunicación social. De este modo, las creencias ayudan al individuo a adaptarse a la realidad en que se encuentra inserto, puesto que éstas se establecen en gran medida como normas que se materializan en el transcurso de la interacción social y en el entorno en que esta interacción se lleva a cabo (Carr y Kemmis, 1988; Morales et al., 1994).

Muchas veces las personas elaboran falsas creencias. Éstas, aún cuando son erróneas, se resisten a una contrastación con la realidad, incluso en ocasiones nos llevan a actuar de formas que producen aparente confirmación. Por ello, nuestras creencias con respecto a otras personas pueden convertirse en profecías que se cumplen a sí mismas. Snyder (1984) demostró que una vez que las creencias erróneas acerca del mundo social se consolidan, pueden inducir a los demás a confirmarlas. Este fenómeno se conoce como "confirmación conductual". Esto implica que las creencias sociales pueden llegar a ser autoconfirmatorias, no sólo en relación con los que nos rodean, sino también en relación a nosotros mismos. En este sentido, las creencias no responden a un conocimiento convencional, por lo que no requieren demostración empírica, ya sea porque la convicción del sujeto que la adopta es tan fuerte que no admite duda o porque su demostración empírica no es posible (Woods, 1996).

En el caso del profesor, las nociones que sustente sobre la educación, sobre los contenidos que imparte, sobre las características del currículum y las condiciones en las que diariamente desarrolla su trabajo, le llevarán a entender, interpretar y tomar decisiones que afectarán su acción en la práctica pedagógica. De ahí la importancia de estudiar las creencias de los profesores (Morales, 2001), pues éstas se entrelazan con la experiencia profesional y se objetivan en el contacto con la realidad escolar. De este modo, las creencias, definidas como constructos personales, muestran una comprensión de las prácticas de los profesores, pues éstos interpretan el proceso educativo desde sus propias creencias. A través de éstas, los profesores perciben, organizan y procesan la información y toman las consiguientes decisiones respecto de su trabajo profesional, puesto que constituyen un factor poderoso en la determinación de sus conductas y demuestran tener gran incidencia en la calidad de los aprendizajes de los estudiantes (Fang, 1996; Duran, 2001; Pajares, 1992).

La investigación en torno a las creencias de los profesores ha demostrado que en el saber pedagógico están comprometidas racionalidades, representaciones y afectos, que son las fuerzas que guían y dan sentido a la práctica pedagógica (González, 1999). De esta forma, los profesores interpretan la situación de enseñanza a la luz de sus creencias acerca del proceso educativo (Morales, 2001). Así, las creencias que sustentan los profesores son empleadas para la organización y desarrollo de su trabajo, y su efecto es observable a través de los objetivos que se proponen, las clases que diseñan, el tipo de interacción que establecen con los alumnos, con el saber que enseñan y, especialmente, con el modelo educativo al cual se adscriben (Sánchez, 2001). 
En la práctica, estas creencias pueden tomar muchas formas. Ellas pueden ser incluidas en las expectativas del profesor sobre el desempeño del estudiante o en las teorías del profesor sobre un área o tema particular de lo que está enseñando (Fang, 1996).

Estas creencias son construidas por los profesores de manera inconsciente a partir de muchos factores. Entre ellos se encuentran: la influencia del significado cultural de la disciplina, la calidad de la experiencia de servicio en las aulas y la oportunidad para la reflexión y la experiencia de servicio (Duran, 2001; Fang, 1996; Marcelo, 2002). Estas creencias se entremezclan con los nuevos conocimientos y modelos prácticos adquiridos, afectando el logro de los resultados educativos esperados. La capacidad para reflexionar sobre estas creencias, confrontándolas con la realidad de su quehacer educativo o con las acciones que éste exige, es una señal positiva de calidad docente. Para el profesor o profesora, como para todo profesional, es importante, por tanto, su capacidad de confrontar las creencias y convicciones que preceden a la ejecución de sus tareas (Ávalos, 2003). Por este motivo es que actualmente, una de las principales áreas de la investigación educacional la constituye las creencias de los docentes, actores fundamentales del proceso educativo (Pajares, 1992).

Desde hace más de una década, a nivel internacional, los estudios han focalizado su atención sobre cómo las convicciones y creencias de los profesores afectan su modo de procesar las demandas educativas. Los profesores, y eso incluye a los futuros profesores, adoptan convicciones y creencias sobre lo que es enseñar; sobre posibilidades o factores que inhiben la enseñanza; sobre tipos de alumnos y sus capacidades; sobre estrategias didácticas y sobre contenidos curriculares y otras materias relacionadas (Rival, 2007).

Considerando lo señalado, resulta indispensable entender la influencia que tienen las creencias en las percepciones y juicios que los profesores realizan y que afecta sus conductas en la sala de clases. Comprender la estructura de las creencias de los profesores y de los candidatos a profesor, también es importante para mejorar la preparación profesional y las prácticas de enseñanza. Además, las creencias constituyen un factor poderoso en la determinación de las conductas del profesor y en su aceptación o rechazo de las propuestas innovadoras (Morales, 2001).

Sin duda, los aspectos señalados, reafirman la noción de que las creencias de los profesores tienen relación con distintos aspectos del proceso educativo y constituyen un punto importante a considerar (González, 1999), aun más si se toma en cuenta que éstas pueden ejercer una influencia importante en el desempeño académico de los educandos.

Rodrigo, Rodríguez y Marrero (1993), sintetizan claramente el impacto de las creencias del educador en el proceso educativo, señalando que un profesional no se modifica en forma sustantiva con sólo mejorar sus conocimientos y la calidad de su saber-hacer, si estas dos dimensiones no son asumidas e interpretadas en un "saber ser" profesional distinto, que les da sentido a las otras dos dimensiones.

\section{SÍNTESIS Y CONCLUSIONES}

Como es posible apreciar, desde hace ya algún tiempo las diferentes investigaciones en el ámbito educacional han mostrado resultados que revelan la función del profesor en el medio escolar, especialmente respecto de aquello referido a su rol como agente de cambio. El rol que se le confiere en momentos de innovación, ha determinado que su 
postura ante los procesos de cambio y mejora represente un fuerte condicionante, sino determinante, del éxito. Esto convierte al profesor en un elemento clave para lograr los resultados esperados (González, 1999). Desde esta perspectiva, la expectativa en relación con el profesor rural es que sea un profesional con una amplia gama de competencias, que sepa cómo decidir e implementar lo que conviene en cada situación de enseñanzaaprendizaje, que tenga conocimiento de la diversidad cultural, adentrándose en el contexto en el cual va a desarrollar su trabajo docente, como una forma de apreciar la cotidianidad en el que se mueven sus estudiantes y rescatar los valores culturales presentes en este contexto, ubicando el desarrollo de la enseñanza en un medio social que otorgue sentido al alumno; esto supone la introducción de estrategias en el proceso de enseñanza-aprendizaje basado en la inducción, interdisciplinariedad, trabajo grupal, apertura social, etc. (San Miguel, 2005; Thomas, 2005). Hoy se espera que el profesor rural sea innovador en sus prácticas, fomente la curiosidad y estimule el conocimiento, desarrollando el pensamiento de sus alumnos, la formación valórica y las habilidades intelectuales e instrumentales básicas de éstos (Arancibia, Herrera y Strasser, 2000).

Lo anterior permite evidenciar el papel central que desempeña el profesor en el proceso de enseñanza y aprendizaje. Por este motivo, se han desarrollado múltiples investigaciones en relación a cuáles serían las posibles implicancias que tienen las características personales del profesor en la calidad de la educación que se ofrece a los niños y niñas que asisten a una escuela. Dentro de esas características personales se encuentran las creencias, cuyo estudio ha cobrado gran relevancia en este último tiempo, debido a que actúan como filtros en la forma en cómo los sujetos perciben el entorno que los rodea y con el cual se relacionan, y que son empleadas para dar sentido a las situaciones (Myers, 2000). En el caso de los profesores, las creencias que tienen sobre el proceso de enseñanza y aprendizaje y sobre sus alumnos, determinan las decisiones pedagógicas que pueden tomar en el aula y que influirán en la calidad de los aprendizajes obtenidos por éstos (Alonqueo, 2001). La investigación ha demostrado que en el saber pedagógico están comprometidas racionalidades, representaciones y afectos, que son las fuerzas que guían y dan sentido a la práctica pedagógica, afectando los resultados de aprendizaje (Sánchez, 2001).

Se podría inferir entonces, a partir de lo mencionado, que cualquier intento por mejorar la calidad del proceso de enseñanza aprendizaje que se lleva cabo en las zonas rurales de nuestro país, que pase por alto el impacto de las creencias del profesor en el proceso educativo y, por ende, en los resultados de aprendizajes de los niños y niñas que asisten a las escuelas rurales de nuestro país, no tendrá los resultados esperados. Esto principalmente debido a que las creencias afectan el comportamiento docente, determinando la forma en que éste conduce el proceso educativo dentro del aula (Contreras y Prieto, 2008).

En este sentido, la importancia de investigar las creencias de los profesores en el medio rural, radicaría principalmente en que a partir de ellas, se podrían inferir muchos de los problemas relacionados con el rendimiento académico evidenciado en las zonas rurales, los que podrían tener relación con creencias de profesores que dan cuenta de bajas expectativas hacia los estudiantes o relacionadas con modelos educativos tradicionales y poco acordes con lo que se necesita actualmente para dar respuesta a un mundo rural cambiante, entre otras. Esto resulta de gran importancia, sobre todo, si se considera que los resultados de aprendizaje de los estudiantes, evidenciados a partir de la aplicación del último SIMCE a escuelas rurales de nuestro país, mostraron una vez más puntajes 
por debajo de lo esperado (SIMCE, 2009). Dichos resultados demuestran que nuestro sistema educativo en zonas rurales, no está respondiendo al objetivo permanente de la educación chilena, es decir, la mejora continua de la calidad de los procesos de enseñanza y aprendizaje, sobre todo, de aquellos que se llevan a cabo en las zonas menos favorecidas de nuestro país.

Lo anterior, por tanto, exige que la investigación sobre creencias sea ampliada hacia el ámbito de la educación rural, permitiendo identificar y describir las creencias que los profesores rurales sustentan acerca del proceso educativo. Esto permitiría generar un perfil del profesor rural que facilitaría, el diseño de políticas educativas propias para la educación rural, que consideren las particularidades del entorno y las múltiples demandas que éste implica para el profesor que se desempeña en estas zonas, y para las cuales, la mayoría de ellos no se encuentra formado. Este perfil permitiría, además, diseñar capacitaciones y perfeccionamientos acordes a lo que hoy se espera del profesor rural, es decir, un profesor con un alto grado de capacidad de actuación y de reflexión sobre su práctica, de adaptabilidad a las situaciones conflictivas y cambiantes de aula y del contexto social rural (Imbernón, 1994). Quizás ésto permitiría terminar con los resultados que numerosos estudios han evidenciado respecto al impacto de los cursos de capacitación y actualización docente, en donde se ha demostrado que el perfeccionamiento no siempre ha sido eficaz para promover y mantener los cambios deseados. Estas estrategias muchas veces son poco pertinentes a las necesidades que los profesores presentan, debido a las particularidades de los contextos en donde ejercen la práctica educativa (Morales, 2001).

Por otra parte, permitir que las creencias sean evidenciadas de forma conciente por los propios profesores genera una oportunidad de reflexión que les facilitaría conocer desde dónde diseñan y llevan a cabo sus prácticas como docentes y cuáles son las influencias que éstas pueden tener en los resultados educativos de los niños y niñas con los cuales trabaja. Esta reflexión requiere que los profesores desarrollen un proceso de autoevaluación y revisión que se mantenga a través del tiempo (Contreras y Prieto, 2008), que permita reorientar el curso de la enseñanza y el aprendizaje, implementando las acciones debidas, en las ocasiones que resulte necesario (Martínez, 2004). Sólo la reflexión sobre el propio ejercicio profesional permite la trasformación del quehacer docente en el aula, toda vez que esto implica repensar la práctica pedagógica, tanto en su sentido como en sus efectos. Esto podría favorecer que las prácticas pedagógicas se orientaran a realizar actividades con alto valor significativo, lo que haría que los niños y niñas de zonas rurales sientan agrado por aprender y sea posible reducir los índices de bajo rendimiento y deserción escolar, que han caracterizado por años a este particular contexto de nuestra educación.

Por último, sería altamente relevante que se realizaran investigaciones sobre las creencias en la educación rural, por el aporte que sus resultados pudiesen tener para focalizar aún más la formación inicial docente, pues saber lo que ocurre en el medio rural, permitiría preparar mejor a los futuros profesores que se desempeñarán ámbito. Esto implica considerar, en los aspectos curriculares de formación, espacios para el desarrollo de procesos reflexivos constantes, que permitan a los futuros profesores ingresar a su mundo de concepciones, teniendo claro cómo éstas incidirán en su actuar como facilitadores del proceso educativo en zonas vulnerables.

Todo lo anterior podría contribuir a dar respuesta a uno de los principales objetivos del modelo educativo actual: responder a la diversidad de sus actores, tanto en lo cultural, como en lo social y étnico. 


\section{REFERENCIAS BIBLIOGRÁFICAS}

Alonqueo, P. (2001). Creencias acerca de la diversidad de género en profesores de enseñanza media municipalizada. Tesis para optar al grado de Magíster en Psicología. Temuco: Universidad de La Frontera.

Arancibia, V., Herrera, P. y Strasser, K. (2000). Manual de Psicología Educacional. Santiago, Chile: Ediciones Universidad Católica de Chile.

Atchoarena, D. y Gasperini, L. (2004). Educación para el desarrollo rural: hacia nuevas respuestas de política. Estudio conjunto realizado por la FAO y UNESCO. Santiago: FAO-UNESCO.

Ávalos, B. (2003). La Formación Inicial Docente en Chile. Santiago de Chile: UNESCO

Canales, M. (2005). La nueva ruralidad en Chile: apuntes sobre subjetividad y territorios vividos. En PNUD-GABINETE, Gobierno de Chile, Seminario Chile rural: Un Desafío para el Desarrollo Humano. Temas de Desarrollo Humano Sustentable, n. 12, 33-39.

Carr, W. y Kemmis, S. (1988). Teoría Crítica de la Enseñanza. Barcelona: Editorial Martínez de Roca.

Contreras, G., Prieto, M. (2008). Las concepciones que orientan las prácticas evaluativas de los profesores: un problema a develar. Estudios Pedagógicos, v. 34, n. 2, 245-262.

Cox, C. (2003). Políticas educacionales en el cambio de siglo. Santiago, Chile: Universitaria.

De Andraca, A.M. y Gajardo, M. (1992). Docentes y Docencia. Las Zonas Rurales. Santiago, Chile: UNESCO/FLACSO.

Delandshere, G. y Jones, J. (1999). Elementary teachers' beliefs about assessment in mathematics: a case of assessment paralysis. Journal of Curriculum and Supervisión, v.14, n.3, 216-240.

De Pujadas, G. (1991). Calidad de la educación. Los nuevos educadores. Santiago de Chile: C.P.U.

De Miguel, A. (2001). Modelos académicos de evaluación y mejora en la Enseñanza Superior. Revista de Investigación Educativa, v.19, n.2, 397-407.

Dutchasky, S. (1999). La escuela como frontera. Buenos Aires: Editorial Paidós.

Duran, E. (2001). Las creencias de los profesores: un campo para deliberar en los procesos de formación. Acción Educativa. Revista Electrónica del Centro de Investigaciones y Servicios Educativos, vol. 1, n.1. Disponible en http://uas.uasnet.mx/cise/rev/Num1.

Fang, Z. (1996). A review of research on teacher beliefs and practices. Educational Research, vol. 38, n.1, 47-64.

Feu i Gelis, J. (2004). La escuela rural en España: apuntes sobre potencialidades pedagógicas, relacionales y humanas de la misma. Revista Digital eRural, Educación, Cultura y Desarrollo Rural. Disponible en http:// http://educación.upa.cl/revistaerural/erural.htm

González, M. (1999). Creencias, atribuciones y autoeficacia en profesores de enseñanza básica municipalizada. Tesis presentada para optar al grado de Doctor en Ciencias de la Educación. Santiago de Chile: Pontificia Universidad Católica de Chile.

Goodson, I. \& U. Numan (2002). Teacher's life worlds, agency and policy contexts. Teachers and Thinking: Theory and Practice, vol. 8, 269-277.

Hashweh, M. (2005). Teacher pedagogical constructions: a reconfiguration of pedagogical content knowledge. Teachers and Thinking: Theory and Practice, v. 11, 273-292.

Imbernón, F. (1994). La formación del profesorado. Buenos Aires: Paidós.

Jackson, P. (2002). Práctica de la enseñanza. Buenos Aires: Amorrortu.

Litwin, E. (2005). La evaluación: campo de controversias y paradojas o un nuevo lugar para la buena enseñanza. En A. Camilloni et al., La evaluación de los aprendizajes en el debate didáctico contemporáneo (pp. 11-34). Buenos Aires: Paidós.

Marcelo, C. (2002). Aprender a enseñar para la sociedad del conocimiento. Education Policy Análisis vol. 10, n. 35. Disponible en http://epaa.asu.edu/epaa/v10n35/

Martínez, R. (2004). El sentido de la evaluación en Educación Básica. Revista Mexicana de Investigación Educativa, vol. 9, n. 23, 817-839. 
Meckes, L. (2007). Evaluación y estándares: logros y desafíos para incrementar el impacto en calidad educativa. Revista Pensamiento Educativo, vol. 40, n. 1. Disponible en http://www. cide.cl/Download/Decano/2002_usos_abusos_simce.pdf

Ministerio de Educación, (1998). Programa de Educación Básica Rural. Santiago. Chile: MINEDUC.

Ministerio de Educación, (1999). Evaluación del Programa Educación Básica Rural 1992 1998. División de Educación General Programa de Educación Básica Rural. Santiago, Chile: MINEDUC.

Ministerio de Educación. (2003). El Marco para la buena enseñanza. Centro de Perfeccionamiento, Experimentación e Investigaciones Pedagógicas. Santiago, Chile: CPEIP.

Montero, C. (2001). La escuela rural: modalidades y prioridades de intervención. Lima: Ministerio de Educación del Perú.

Morales, J., Moya, M., Rebolloso, E., Fernández, J., Huici, C., Marques, J., et al. (1994). Psicología Social. Madrid, España: McGraw-Hill.

Morales, S. (2001). Creencias y valores de educadores de párvulos en la comuna de Temuco. Tesis presentada a la Universidad de la Frontera para optar al grado de Magíster en estudios Psicológicos. Temuco: Universidad de la Frontera.

Myers, D. (2000). Psicología Social. Bogotá, Colombia: Mc Graw Hill.

Navas, L., Sampascual, G. y Castellón, L. (1992). Atribuciones y Expectativas de Alumnos y Profesores: Influencias en el Rendimiento Escolar. Revista de Psicología General y Aplicada. Argentina: Universidad de la Rioja.

Pajares, M. (1992). Teacher's beliefs and educational research: cleaning up a messy construct. Review of Educational Research, vol. 6, n. 3, 307-332.

Programa de las Naciones Unidas para el Desarrollo. (2008). Desarrollo Humano en Chile Rural, 6 millones de nuevos caminos. Santiago, Chile: PNUD.

Rivera, M. y Milicic, N. (2006). Percepciones, Creencias, Expectativas y aspiraciones de Padres y Profesores de enseñanza general básica. Psykhé, vol. 15, n. 1, 119-135.

Rival, H. (2007). El conocimiento estratégico y procedimental implicado en la formación inicial docente. Tesis doctoral. Barcelona: Universitat Ramón LLull.

Rodrigo, M., Rodríguez, A., Marrero, J. (1993). Las teorías implícitas: Una aproximación al conocimiento cotidiano. Madrid: Visor.

Sánchez, T. (2001). Creencias acerca del proceso educativo en alumnas de la carrera de Educación de Párvulos: Universidad Católica de Teтисо. Tesis para optar al grado de Magíster en Estudios Psicológicos. Temuco: Universidad de La Frontera.

San Miguel, J. (2000). Escuela Rural, Historias de Microcentros. Santiago: Pehuén.

San Miguel, J. (2005). Nuevas demandas de la educación básica rural. Revista Digital eRural, Educación, Cultura y Desarrollo Rural. Disponible en http://www.revistaerural.cl/jsm.htm

Schiefelbein, E. (1992). Relación entre la calidad de la educación y el modelo de enseñanza frontal en América Latina. Boletín Proyecto Principal de Educación en América Latina y el Caribe, vol. 29, 3-18.

SIMCE. (2009). Resultados Nacionales SIMCE 2008. Santiago: Ministerio de Educación, Gobierno de Chile. Disponible en http://simce.cl/fileadmin/Documentos_y_archivos_SIMCE/Informes_ Resultados_2008/Informe_Nacional_2008.pdf

Snyder, M. (1984). When belief creates reality. Advances in experimental social psychology Journal, vol. 18, 247-305.

Thomas, C. (2005). El rol del profesor en la educación rural chilena. Revista Digital eRural, Educación, Cultura y Desarrollo Rural. Disponible en http://www.revistaerural.cl/thyhe.htm.

Torrance H. y J. Pryor (2001). Developing Formative Assessment in the Classroom: Using Action Research to Explore and Modify Theory. British Educational Research Journal, vol. 25, n. $5,615-631$. 
Villarroel, G. (2003). El profesor rural de Chiloé. Revista Digital eRural, Educación, Cultura y Desarrollo Rural. Disponible en http://educacion.upa.cl/revistaerural/GLADYSVIa.htm

Villarroel, J. (2004). El Desarrollo Rural en el Chile de hoy, un desafío para la superación de la pobreza. Santiago de Chile: Fundación Nacional para la Superación de la Pobreza.

Williamson, G. (2004). Estudios sobre la educación para la población rural en Chile. En Educación para la población rural en Brasil, Chile, Colombia, Honduras, México, Paraguay y Perú. Proyecto FAO-UNESCO-DGCS/ITALIA-CIDE-REDUC. Roma: FAO-UNESCO.

Woods, D. (1996). Teacher cognition in language teaching: beliefs, decision-making and classroom practice. Cambridge: Cambridge University Press. 